\title{
Proprioceptive Input to Feeding Motor Programs in Aplysia
}

\author{
Colin G. Evans ${ }^{1}$ and Elizabeth C. Cropper ${ }^{1,2}$ \\ ${ }^{1}$ Department of Physiology and Biophysics and ${ }^{2}$ The Fishberg Center for Research in Neurobiology, The Mount Sinai \\ Medical Center, New York, New York 10029
}

\begin{abstract}
Although central pattern generators (CPGs) can produce rhythmic activity in isolation, it is now generally accepted that under physiological conditions information from the external and internal environment is incorporated into CPG-induced motor programs. Experimentally advantageous invertebrate preparations may be particularly useful for studies that seek to characterize the cellular mechanisms that make this possible. In these experiments, we study sensorimotor integration in the feeding circuitry of the mollusc Aplysia. We show that a premotor neuron with plateau properties, B51, is important for generating the radula closing/retraction phase of ingestive motor programs. When B51 is depolarized in semi-intact preparations, radula closing/retractions are enhanced. When B51 is hyperpolarized, radula closing/retractions are reduced in size.
\end{abstract}

Many rhythmic behaviors are generated by circuits of neurons referred to as central pattern generators (CPGs) (Delcomyn, 1980). Although CPGs can produce motor patterns in isolation, it is now generally accepted that sensory neurons, e.g., proprioceptors, play an important role in generating many physiological motor programs (e.g., Pearson, 1987). Proprioceptor-CPG interactions may influence phase transitions and/or play a role in determining the magnitude of the motor output (e.g., Pearson and Ramirez, 1997). The magnitude of the motor output must increase accordingly when the load on a muscle is increased. This presumably increases activity in proprioceptors, which directly or indirectly excite the motor neurons that innervate the loaded muscle. One mammalian behavior in which this type of compensation has been extensively studied is locomotion. For example, extensor activity is increased when an animal walks up an incline. It is likely that this results, at least in part, from the increased load on extensor muscles and the corresponding increased activity of extensor proprioceptors (Pearson and Ramirez, 1997). Although it has been estimated that peripheral afferents make a significant contribution to locomotion under physiological conditions (e.g., Yang et al., 1991), it has been difficult to establish this directly in vertebrates.

\footnotetext{
Received April 23, 1998; revised July 15, 1998; accepted July 17, 1998.

This work was supported by an Irma T. Hirschl Career Scientist award, Research Scientist Development Award MH-01267, and Public Health Service Grant MH51393. Some of the Aplysia used in this study were provided by the National Center for Research Resources National Resource for Aplysia at the University of Miami under National Institutes of Health Grant RR10294. We thank Drs. Paul Church, Itay Hurwitz, and Klaudiusz Weiss for their valuable comments on an earlier draft of this manuscript.

Correspondence should be addressed to Dr. Elizabeth C. Cropper, Department of Physiology and Biophysics, Box 1218, Mount Sinai School of Medicine, 1 Gustave L. Levy Place, New York, NY 10029.

Dr. Evans's present address: Phase V Communications Inc., 114 Fifth Avenue, New York, NY 10011.

Copyright (ㄷ) 1998 Society for Neuroscience $\quad 0270-6474 / 98 / 188016-16 \$ 05.00 / 0$
}

In addition to being important as a premotor interneuron, B51 is also a sensory neuron that is activated when the feeding apparatus, the radula, rotates backward. The number of centripetal spikes in B51 is increased if the resistance to backward rotation is increased. Thus, B51 is a proprioceptor that is likely to be part of a feedback loop that insures that food will be moved into the buccal cavity when difficulty is encountered. Our data suggest, therefore, that Aplysia are able to adjust feeding motor programs to accommodate the specific qualities of the food ingested because at least one of the neurons that generates the basic ingestive motor program also serves as an on-line monitor of the success of radula movements.

Key words: proprioceptive input; Aplysia; central pattern generator; load compensation; plateau potentials; feeding behavior

Proprioceptive input is also important for rhythmic behavior in invertebrates. Invertebrate preparations have experimentally advantageous features that have made it possible to record from neurons in intact animals engaged in normal behavior (e.g., Wolf and Pearson, 1988). This work has more clearly established that proprioceptor-CPG interactions are in fact important under physiological conditions. Although this has been most extensively documented for arthropods, it is also likely to be true in molluscs (e.g., Jahan-Parwar et al., 1983). Proprioceptor-CPG interactions have, however, been less extensively investigated in molluscan preparations, despite the fact that the molluscan nervous system is likely to be particularly advantageous for studies of current interest in this field, i.e., studies that characterize synaptic mechanisms important for sensorimotor integration. For example, sensory neurons in molluscs can be centrally located and accessible for electrophysiological manipulation and biochemical characterization (e.g., Rosen et al., 1992, 1993, 1994; Miller et al., 1994; Cropper et al., 1996).

In these experiments, conducted in the feeding circuitry of Aplysia, we demonstrate that a centrally located premotor neuron with plateau properties, B51 (Plummer and Kirk, 1990), is a proprioceptor. We characterize the peripheral mechanism that generates centripetal activity in B51 and demonstrate that centripetal spikes in B51 have to coincide with a rhythmic central depolarization if they are to be effective at driving follower neurons. We also show that centripetal spikes are not generated in B51 during all feeding motor programs. They are particularly apparent during swallowing-like responses when an object is moved into the buccal mass. Finally, we provide evidence that changes in B51 activity are in fact likely to have an impact on radula movements. Thus, B51 is not only a proprioceptor but also an interneuron whose activity strongly impacts buccal motor programs. 
Parts of this paper have been published previously (Evans and Cropper, 1997).

\section{MATERIALS AND METHODS}

Animals. Aplysia californica $(200-400 \mathrm{gm})$ were maintained at $14-16^{\circ} \mathrm{C}$ in 150 gallon holding tanks containing aerated artificial seawater (ASW). In all experiments, animals were anesthetized with isotonic magnesium chloride $(50 \% \mathrm{w} / \mathrm{v})$.

Identification of neurons. B51 was initially identified as described by Plummer and Kirk (1990); i.e., it has plateau properties, and a number of its synaptic connections have been characterized. In addition, we routinely filled B51 with 5(6)-carboxyfluorescein dye (see below) to verify its anatomy and characterized additional synaptic connections that are described in this report.

B44 was originally identified in an isolated buccal mass preparation in which a cut was made through the pharynx to expose the radula halves. The esophageal nerves and buccal nerve 1 were cut, and the buccal ganglion was twisted slightly to expose its caudorostral surface. B44 was identified using three criteria. (1) When it was depolarized, it caused a bilateral contraction of the inner I4 leaflets and a backward rotation with slight opening of the radula. (2) This contraction evoked centripetal spikes in B51. (3) B44 was filled with carboxyfluorescein, and its anatomy was characterized. The neuron identified in this manner was then confirmed as being the cell described by Church and Lloyd (1994) (P. Church, personal communication).

B8 motor neurons were identified on the basis of morphology [as determined by injection of carboxyfluorescein (see below)] and position and their ability to cause the radula to close (Gardner, 1971; Church and Lloyd, 1991; Morton and Chiel, 1993b).

Characterization of the innervation of the I4 muscle complex. An isolated buccal mass with the buccal ganglion attached was pinned ventral side up in a Sylgard-lined dish. The buccal ganglion was pinned to expose the caudorostral surface, and the I 2 muscle was trimmed to expose the I4 muscle. A cut was then made from the jaws, splitting I6 so that the inner leaflets of the I4 muscles were exposed. This permitted access for intracellular recording from muscle fibers.

To measure length changes of the I4 muscles, we reduced the buccal mass further so that it consisted of the radula sac, the I4 muscles, and the intact radular nerve. Contractions were transduced using previously described methods (Evans et al., 1996). Briefly, a wooden beam was attached at its midpoint to the rotating arm of a semi-isotonic force transducer (the transducer shown in Fig. 1; Harvard Apparatus), which served as a movement detector. One end had a metal hook to which the freed end of the I4 muscle was attached by means of a silk suture. The other half of the beam was marked with a centimeter scale, along which a known weight could be moved to vary the load on the muscle. A stop was placed under the wooden beam to control muscle length.

In experiments in which synaptic transmission in the nervous system was selectively abolished, the buccal ganglion was encircled by a ring of Vaseline, which "sealed" a Lucite subchamber in place. The ASW bathing the buccal ganglion could then be specifically exchanged while the periphery remained in normal ASW.

The carbachol preparation. These experiments were conducted in a modified version of a preparation developed by Susswein et al. (1996). Specifically, an isolated buccal mass with the buccal and cerebral ganglia attached was pinned to a Sylgard dish. Two pins were inserted through the jaws on either side, to prevent them from closing. No other pins pierced the buccal mass, although a pin was pushed into the Sylgard on either side of the buccal mass to prevent it from rolling during motor programs. All buccal nerves were intact except for the esophageal nerve. The buccal and cerebral ganglia were pinned to a raised Sylgard platform, and the cerebral ganglion was isolated in a Lucite chamber sealed onto the Sylgard with Vaseline. The esophagus was removed, and a hole was cut in the pharynx to expose the radula halves. Motor programs were elicited by applying $10^{-3} \mathrm{M}$ carbachol directly to the cerebral ganglion, which was not desheathed.

To monitor movements of the buccal mass, we tied a silk suture to the anterior tip of the radula (Fig. 1). The thread was then pulled through the jaws and attached to the semi-isotonic force transducer described above (Harvard Apparatus). In these experiments this transducer detected movement of the radula toward the jaws, referred to as "protraction," and movement of the radula back toward esophageal tissue, referred to as "retraction." Protractions and retractions actually consist of at least two components-forward or backward rotation and protraction or retraction. We use the terms protraction and retraction for simplicity because a
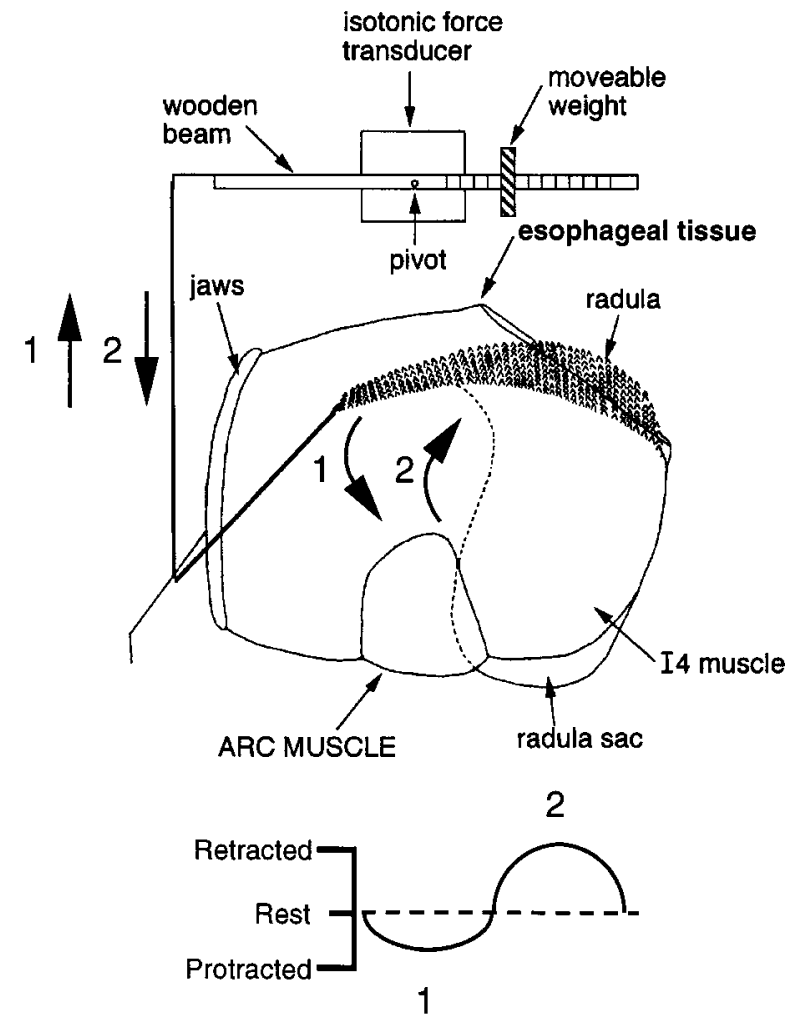

Figure 1. Schematic illustration of the preparation used to transduce movements of the buccal mass. (Ganglia are not shown.) A string was tied to the anterior tip of the radula and attached to a movement transducer. This transducer detects movement of the radula toward the jaws, which is referred to as protraction (arrow 1), and movement of the radula toward esophageal tissue, which is referred to as retraction (arrow 2). Protraction produces a downward deflection in transducer records, and retraction produces an upward movement. The rest position of the radula (the position before movement is elicited) is indicated by the dotted line in the bottom diagram.

detailed analysis of buccal mass movements is beyond the scope of this study (instead see Drushel et al., 1997). As shown in Figure 1, protraction produces a downward deflection in transducer records, and retraction produces an upward movement. The "rest" position of the radula (the position before movement is elicited) is indicated by a dotted line.

Recording. Intracellular electrodes were glass micropipettes filled with a solution of $3 \mathrm{M}$ potassium acetate containing $30 \mathrm{mM} \mathrm{KCl}$. For simultaneous current injection and voltage recording, we used double-barreled electrodes beveled so that they had resistances ranging from 5 to $10 \mathrm{M} \Omega$. Recordings from muscle fibers were made with single-barreled electrodes with resistances of $10-25 \mathrm{M} \Omega$. Preparations were routinely grounded by the use of a chlorided silver wire.

Intracellular dye injection. A $3 \%$ solution of the fluorescent dye 5(6)carboxyfluorescein in $0.1 \mathrm{M}$ potassium citrate, titrated to $\mathrm{pH} 8.0$, was iontophoresed into neurons from single-barreled glass microelectrodes with tips beveled to lower impedances to $\sim 10 \mathrm{M} \Omega$ (see Rao et al., 1986). To reduce active transport of the dye, probenecid (10 mM final concentration) was added to preparations (Steinberg et al., 1987; Rosen et al., 1991), which were kept at $4^{\circ} \mathrm{C}$ for $12-15 \mathrm{hr}$.

Reagents. The ASW used in these experiments had the following composition (in mM): $460 \mathrm{NaCl}, 10 \mathrm{KCl}, 11 \mathrm{CaCl}_{2}, 55 \mathrm{MgCl}_{2}$, and 5 $\mathrm{NaHCO}_{3}$. All salts and carboxyfluorescein were obtained from Sigma (St. Louis, MO).

\section{RESULTS}

\section{B51 as a sensory neuron}

In their original description of its morphology, Plummer and Kirk (1990) noted that B51 has a process in the radular nerve. To visualize this process, we injected B51 with carboxyfluorescein 



Figure 2. Anatomy of B51. A, Camera lucida drawing of two B51 neurons in the same preparation filled with carboxyfluorescein dye. $B$, Semischematic drawing showing the peripheral projection of a B51 neuron. Note that the B51 process can be traced to the black tissue contiguous with the esophagus overlying the I4 muscle complex.



dye $(n=10)$. We found that the axon of B51 remains in the ipsilateral branch of the radular nerve when it divides (Fig. $2 A$ ). From this branch, the axon enters the immediately adjacent tissue of the pharynx/esophagus and can be traced to the black tissue contiguous with the esophagus that overlies the I4 muscle complex (Fig. 2B)

The peripheral branch of B51 does not appear to provide efferent innervation of the I4 muscle. When B51 is stimulated, junctional potentials are not recorded from I4 fibers in the vicinity of the B51 axon. In addition, stimulation of B51 does not elicit discrete, localized contractions of buccal musculature. In the absence of evidence that B51 is a motor neuron, we sought to determine whether it is a sensory neuron. We stretched leaflets of the I4 muscle and found that a series of $20-25 \mathrm{mV}$ potentials, resembling centripetal spikes, was recorded from B51 (Fig. 3).

\section{Innervation of the 14 muscles}

To determine whether physiological contractions of the I4 muscle would activate B51, it was necessary to identify the I4 motor neurons. The I4 muscle complex is not a solid muscle but has a leaflet structure (Scott et al., 1991). More specifically, we found that the I4 complex actually consists of outer leaflets and a set of three inner leaflets (dorsal, medial and ventral) (Figs. 4B1, 5A). The inner leaflets attach along the length of the posterior of the radula sac, partly fusing with each other, and attach anteriorly to the I1/I3 muscle block. The inner leaflets do not attach to esophageal tissue. The inner leaflets are partly overlain by the outer leaflets. The outer leaflets are shorter and thinner than are the inner leaflets and are attached anteriorly to the inner leaflets and posteriorly to esophageal tissue (except for a narrow ventral strip that attaches to the radula sac). Not surprisingly, in view of its gross structural complexity, the I4 muscle complex is innervated by more than one motor neuron. In this study, we concentrated on two neurons (B44 and B8) because they are particularly effective at inducing centripetal activity in B51.

\section{Neuron B44}

Neuron B44 is a large $(200-300 \mu \mathrm{m})$ cell that occupies a slightly off-center position on the caudorostral surface of the buccal ganglion (Church and Lloyd, 1994) (Fig. 4A). It has two axons, one projecting branches into the ipsilateral buccal nerves 2 and 3 and the other sending a bifurcating branch into the radular nerve before crossing the commissure to send further branches along contralateral nerves 2 and 3 . We were not able to unequivocally 


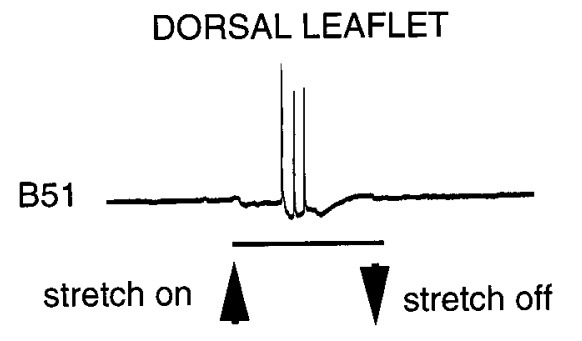

MEDIAL LEAFLET

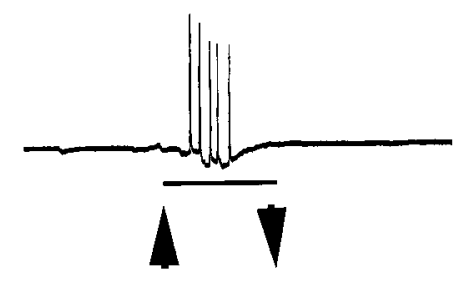

VENTRAL LEAFLET

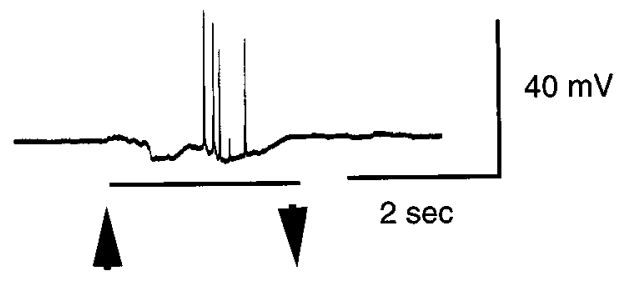

Figure 3. Brief stretches applied to each I4 leaflet (dorsal, medial, and lateral; see Fig. 4B1) evoke what appear to be centripetal spikes in B51.

identify B44 neurons in both buccal hemiganglia in the same preparation. Given that B44 has bilateral projections, there may indeed only be one cell per ganglion.

When B44 is stimulated, spikes are one-to-one with excitatory junctional potentials (EJPs) in each of the ipsilateral and contralateral inner leaflets of the I4 muscle ( $n=7$; Fig. 4 B2, B3) but not in the outer leaflets $(n=6)$. B44 appears to innervate the posterior aspect of the inner leaflets more strongly than the anterior aspect because posterior EJPs are larger than anterior EJPs (Fig. 4B2 vs B3). Interestingly, the posterior I4 leaflets are closer to the tissue in which B51 processes are visualized. Contractions of the I4 muscle evoked by B44 persist when the buccal ganglion is placed in a separate subchamber and bathed in 0 $\mathrm{Ca}^{+2}$ and $10 \mathrm{mM} \mathrm{Co}^{+2} \mathrm{ASW}$ to block synaptic transmission $(n=$ 5). Although this does not eliminate polysynaptic transmission in the periphery, we found that spikes in B44 and EJPs in I4 muscle fibers are reliably one-to-one, with constant delays of 80-100 msec. Contractions of the I4 muscles are elicited by B44 when buccal nerves 2 and 3 are cut, indicating that the axon in the radular nerve innervates this muscle group. (Spikes in B44 also elicit EJPs in other muscles of the buccal mass, e.g., I1. We did not, however, characterize this innervation in more detail because we were interested in neuromuscular units that would activate B51.)

The concerted muscle contractions produced when B44 is stimulated in intact buccal mass preparations evoke two movements, a backward rotation of the radula and a slight opening of the posterior halves of the radula. This opening was not as vigorous as that elicited by B48, the motor neuron that innervates the
I7-I10 muscles (Evans et al., 1996). Additionally, B48-induced radula openings are accompanied by radula protraction (instead of retraction) (Evans et al., 1996).

I4 muscle contractions could be produced by stimulating B44 neurons at frequencies that are observed during rhythmic motor programs. These frequencies are, however, only reached after the radula closing/retraction phase of ingestive motor programs has already begun (e.g., see Fig. $8 A$ in which contractions are elicited when B44 is stimulated at $10-15 \mathrm{~Hz}$ vs Fig. 16 in which B44 eventually fires at $\sim 12 \mathrm{~Hz}$ during the closing/retraction phase of a carbachol-elicited motor program). This observation, taken together with the fact that other motor neurons that are likely to produce radula retraction have been described (e.g., see Church and Lloyd, 1994), suggests that B44 contributes to radula retraction but is not solely responsible for it.

\section{Neuron B8}

The B8 motor neurons, B8a and B8b, are located on the rostral surface of the buccal ganglion (Gardner, 1971) and elicit radula closing (Morton and Chiel, 1993b). When the B8 neurons are stimulated, spikes are one-to-one with EJPs in fibers of the outer I4 leaflets of the I4 muscle $(n=5$; Fig. $5 B)$ but not in those of the inner leaflets $(n=5)$. Cutting the outer leaflets of the I4 muscles does not abolish radula closure induced by B8, so another as yet unidentified muscle group presumably produces this movement.

\section{Central interactions between B51 and the 14 motor neurons and centripetal spikes in B51 as a result of 14 muscle contractions}

After we had identified the I4 motor neurons, we characterized central interactions between B51 and these cells and determined whether motor neuron-induced muscle contractions would elicit centripetal spikes in B51.

\section{Neuron B44}

Neurons B51 and B44 are electrically coupled centrally, with the electrical coupling being more effective in the B51 to B44 direction than vice versa $(n=3$; Fig. 6). This is apparent with hyperpolarization but is particularly clear with depolarizing pulses. The resting potential of B51 is generally $\sim 15-20 \mathrm{mV}$ below threshold. Consequently, when B51 is activated by injecting current, action potentials ride on top of an underlying DC component (see Fig. 6A, arrow). Thus, B51 effectively depolarizes B44 and causes it to spike. In contrast, at its resting potential, neuron B44 is closer to threshold than is B51. Consequently, when B44 is depolarized, it begins to spike and less effectively depolarizes B51 (Fig. 6B). In experiments in which B44 is stimulated to produce I4 contractions and centripetal spikes in B51, the electrical coupling between B44 and B51 is, therefore, often not very apparent.

When B44 is stimulated in intact buccal mass preparations, 20-25 mV excitatory potentials are generally recorded from the soma of B51 ( $n=21$ out of 27 preparations; e.g., Fig. 7). These potentials do in fact occur with a delay after the onset of an I4 muscle contraction (Fig. 7A1). Moreover, they continue to be evoked after the current being injected into B44 is switched off. Several types of data confirm that B44-elicited potentials in B51 are in fact centripetally elicited. Namely, potentials are decreased in amplitude when B51 is hyperpolarized (Fig. 7C). Additionally, potentials are abolished when the buccal nerves are cut $(n=2$; Fig. 7A2) but do persist when buccal ganglia are placed in a separate subchamber and are bathed in $0 \mathrm{Ca}^{+2}$ and $10 \mathrm{mM} \mathrm{Co}^{+2}$ $\operatorname{ASW}(n=5$; Fig. $7 B)$ (which abolishes synaptic transmission). 

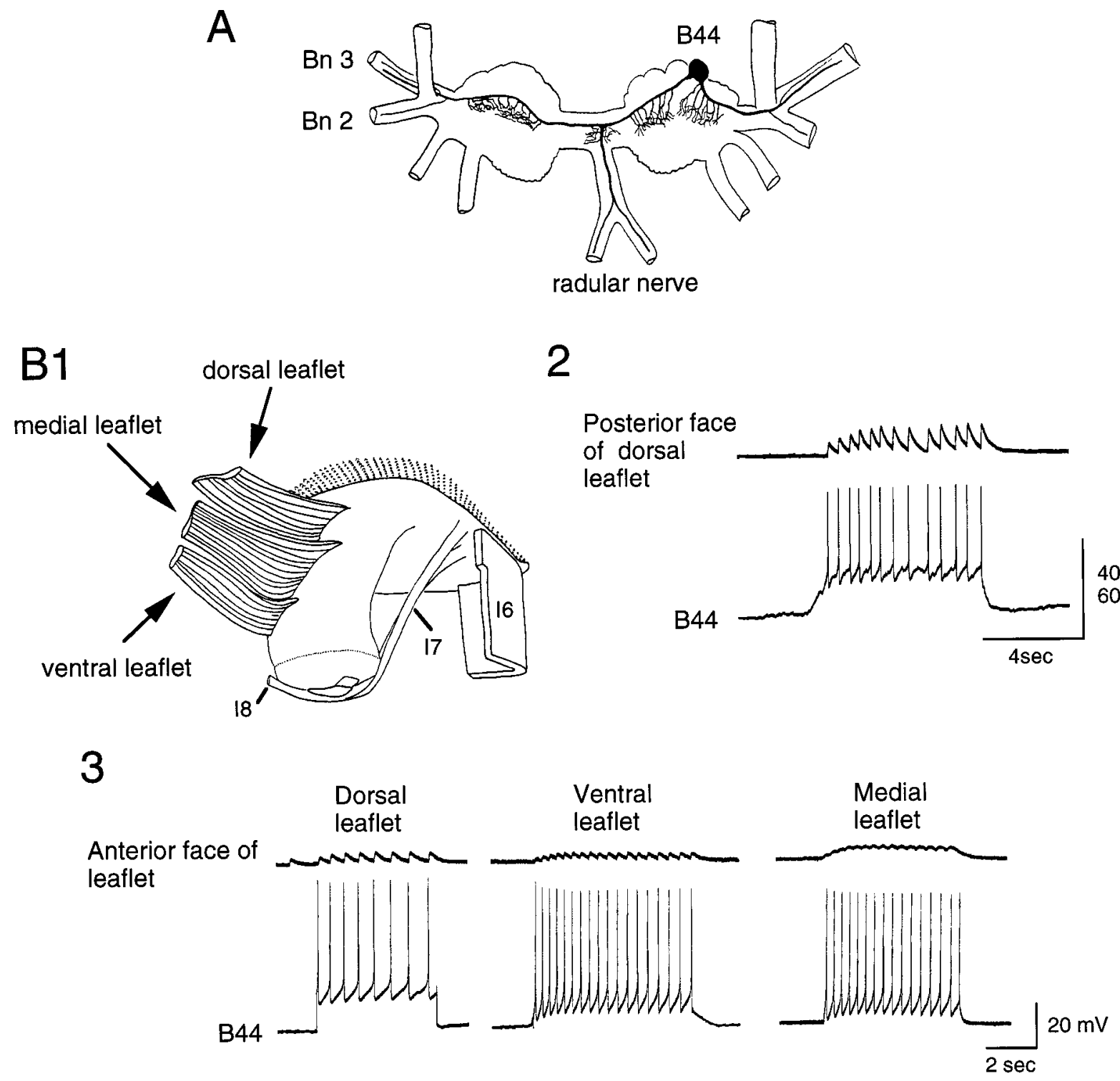

Figure 4. B44 innervates the inner leaflets of the I4 muscle complex. A, Camera lucida drawing of B44 is shown. Bn 2 and Bn 3 , Buccal nerves 2 and 3. B1, Semischematic drawing of the radula and attached musculature is shown. The I4 muscle complex has been flipped back to expose the inner leaflets. B2, Intracellular stimulation of B44 (bottom) elicits EJPs recorded intracellularly from a fiber of the dorsal inner leaflet (top). More specifically, EJPs were recorded from the posterior side of the dorsal leaflet, i.e., the side that is closest to the outer leaflets. B3, Stimulation of B44 (bottom) also elicits EJPs that can be recorded from the anterior face of the inner leaflets (top), i.e., the side shown in B1. Note that EJPs recorded from the posterior face of the inner leaflets are larger than those recorded from the anterior face. The posterior face of the inner leaflets lies directly under the tissue that contains processes of B51.

To determine whether the number of centripetal spikes evoked in B51 is related to parameters of muscle contractions, we conducted experiments in reduced neuromuscular preparations and increased contraction size by increasing the firing frequency of B44. The number of centripetal spikes elicited in B51 also increased (Fig. $8 A$ ). In other experiments, which were performed in semi-intact preparations, we increased the resistance to backward rotation by tying a string to the anterior tip of the radula and holding this string as B44 was fired. The number of centripetal spikes evoked in B51 was also increased by this manipulation $(n=$ 6 out of 7 preparations; Fig. $8 B$ ). This latter phenomenon was further investigated in the reduced preparation in which I4 contractions could be transduced. The number of centripetal spikes evoked in B51 was increased when the counterweight on the transducer was increased $(n=2$; Fig. $8 C$ ). Thus, B51 seems to function as a proprioceptor that detects the load on the I4 muscle.

\section{Neuron B8}

There is generally no obvious electrical coupling between B51 and the B8 neurons. When B51 is stimulated, EPSPs are recorded from the B8s, one-to-one with B51 action potentials $(n=9$; Fig. $9 A)$. These EPSPs seem to be chemical because they are abolished in $0 \mathrm{Ca}^{+2}$ and $10 \mathrm{~mm} \mathrm{Co}{ }^{+2}$ solutions $(n=2$; Fig. 9A2). They do not, however, always become larger when B8 is hyperpolarized, as might be expected for a conventional chemical connection. Interestingly, however, the conductance of B8 increases with hyperpolarization (A. Klein, personal communication). This inward rectification may, therefore, act to reduce the 


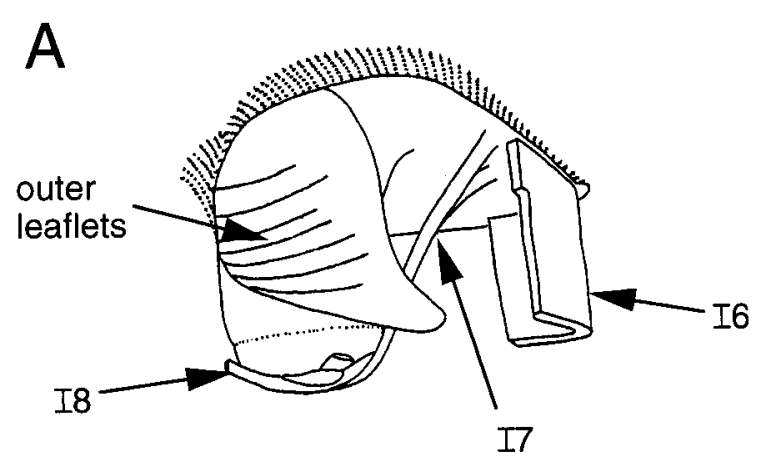

B
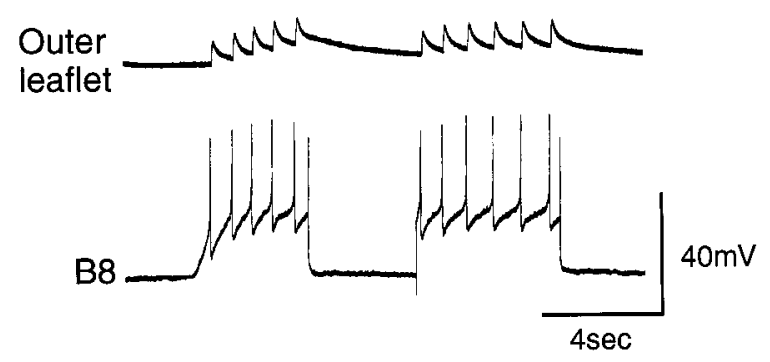

Figure 5. B8 innervates the outer leaflets of the I4 muscle complex. $A$, Semischematic drawing of the radula and some of its attached musculature, including the I4 muscle complex, is shown. The outer leaflets of the I4 complex are visible because they overlie the inner leaflets. $B$, Intracellular stimulation of B8 (bottom) elicits EJPs recorded intracellularly from fibers of the outer leaflets (top).

size of postsynaptic potentials (PSPs) (see Kandel and Tauc, 1966). Thus, although the B51-B8 connection seems to be chemical in nature, a definitive characterization of this synapse will not be possible until the biophysical properties of B8 have been characterized in detail. In any case, direct connections between the B51 and the B8 neurons only operate effectively in one direction. When B8 is stimulated in preparations in which the peripheral musculature is not present, direct chemical responses are not evoked in B51.

When B8 is stimulated in semi-intact preparations, however, centripetal spikes are recorded in B51 in some preparations (i.e., $n=13$ out of 22 preparations; Fig. 9B). Centripetal activity in B51 elicited by stimulating B8 is, however, generally much less robust than is activity elicited by B44 in that with repeated trials B8 becomes progressively less effective at evoking B51 responses. The number of centripetal spikes evoked by B8 can be increased by placing an object between the radula halves and increasing the resistance to radula closing ( $n=2$ out of 4 preparations; Fig. 9B).

\section{Central gating of afferent activity in B51}

Many of the follower neurons of B51 are cells that are electrically coupled to it (e.g., Fig. 6) (e.g., Plummer and Kirk, 1990). Because centripetal spikes are relatively fast events, they are obviously attenuated as they are transmitted to coupled cells. Moreover, because some of the follower neurons of B51 are not close to threshold when they are at their resting membrane potential (e.g., B15), afferent activity in B51 generally does not cause these neurons to spike. Thus, when B51 is at its resting membrane potential, afferent activity in B51 is generally not very effective at driving electrically coupled follower neurons. It is also not very effective at driving B8 (Fig. 10A,C). As is shown in Figure 9A1, when B51 is activated by intracellular current injection, PSPs are recorded in B8. Under these conditions, however, there is a significant underlying depolarization of the somata of B51. In contrast, however, when B51 is activated peripherally, PSPs are not recorded in $\mathrm{B} 8$ (Fig. $10 A, C$ ). Thus, in a quiescent buccal ganglion, afferent activity in B51 is not likely to effectively drive its follower neurons.

When B51 is centrally depolarized, however, centripetal spikes do become effective at driving follower neurons. This is shown in Figure 10, which is an experiment in which centripetal spikes are evoked in B51 by stimulating B44 at physiological frequencies under two conditions, i.e., when B51 is at its resting membrane potential (Fig. 10A,C) and when B51 has been depolarized by 15 $\mathrm{mV}$ (Fig. 10B). PSPs are only recorded in B8 when B51 is centrally depolarized. Obviously this paradigm could be repeated for each of the other B51 followers. Because, however, at least eight of these cells have been identified [i.e., B3, B9, B15, B16, B4, B5, B44, B8 (Plummer and Kirk, 1990; Figs. 6, 9)] and more are likely to exist, we conducted our next experiments in a semi-intact buccal mass preparation in which movements of the buccal mass could be transduced $(n=3)$. Thus, with this paradigm, we used movements of the buccal mass as a general indicator of activity in buccal motor neurons. When B44 stimulation elicited centripetal spikes in B51 when it was at its resting potential, movements of the buccal mass were relatively weak (Fig. 11 $\mathrm{A}$, left). (Presumably they were a direct result of the B44-evoked contraction.) B51 was then depolarized, and centripetal spikes were again elicited via B44-evoked contractions relatively soon after the depolarization (Fig. 11 $A$, middle). Centripetal spikes now triggered a plateau in B51 that elicited spikes in B44 (see bracket in Fig. 11A, middle) and elicited a more vigorous radula retraction (Fig. 11 $A$, middle, right). In Figure $11 B$, we performed a similar experiment except that B51 was held at depolarized potentials for a longer time before centripetal spikes were triggered. With this paradigm, it is more obvious that depolarizations alone are not sufficient to trigger plateaus in B51. Thus, these experiments indicate that afferent activity in B51 is only likely to be effective at driving follower neurons if B51 is centrally depolarized.

\section{B51 is centrally depolarized during ingestive motor programs}

To determine whether B51 does in fact receive central input during ingestive motor programs, we performed experiments in a modified version of a preparation developed by Susswein et al. (1996). Video analysis has indicated that programs initiated by applying carbachol to the cerebral ganglion in these preparations are ingestive [as opposed to egestive (Susswein et al., 1996)]. Our results confirmed this because in most cases radula opening and protraction occurred together, as did radula closing and retraction. [Radula closing occurs during radula protraction when animals make egestive responses (Morton and Chiel, 1993a).] Additionally, the accessory radula closer (ARC) motor neuron B15 was active during carbachol-elicited motor programs $(n=4$; Fig. 12). When intact animals make egestive responses, neuron B15 does not fire (Cropper et al., 1990). We modified the preparation developed by Susswein et al. (1996) in that we transduced movements of the buccal mass.

In the majority of preparations ( 8 out of 15 ), carbachol induced a motor program characterized by a weak protraction of the radula from the rest position toward the jaws and then a retraction back to the rest position or slightly beyond rest position (e.g., Fig. 12; Fig. 13, middle panel). This type of activity most closely 
A Current Injected into B5I

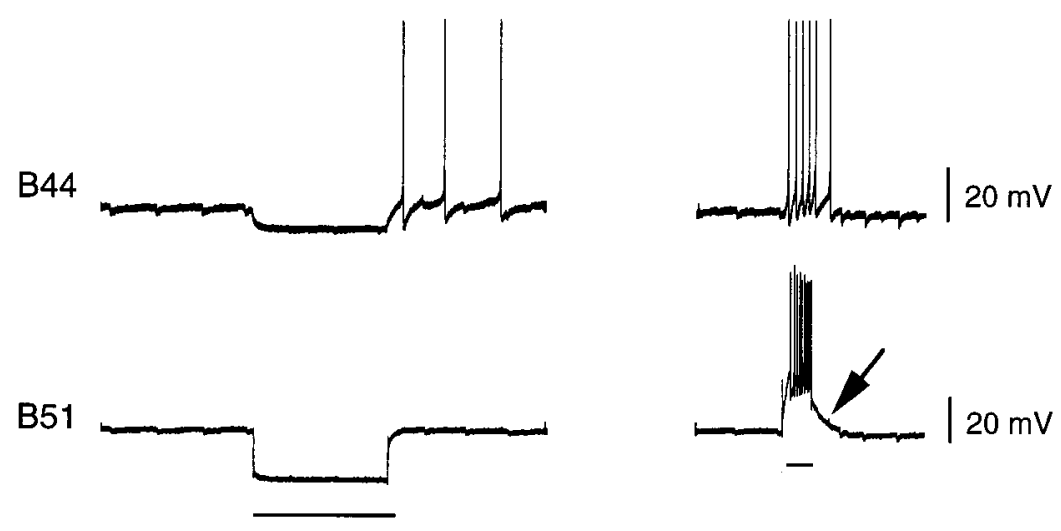

B $\quad$ Current Injected into B44



Figure 6. Experiment done in an isolated buccal ganglion showing that B44 and B51 are electrically coupled. Current injections are indicated by horizontal bars. When B51 is depolarized $(A)$, it is more effective at driving B44 than vice versa $(B)$. In part this is attributable to the fact that B51 is further from threshold when it is at its resting membrane potential. Consequently, when B51 is activated by injecting current, there is an underlying DC component to evoked activity (arrow in $A$ ). corresponds to biting-like behavior (as opposed to swallowinglike behavior, the alternative type of ingestive response). Programs are more biting-like than swallowing-like because the radula does not fully retract to reach the esophagus. In intact animals, biting occurs when animals make consummatory responses but do not successfully ingest food (Kupfermann, 1974). The radula does not fully hyper-retract because there is no food to be deposited in the esophagus (Fig. 14). We refer to responses as biting-like because movements of the buccal mass are not identical to those observed in intact animals. Most noticeably, the radula was never extended through the jaws as it is in intact animals (Kupfermann, 1974; Drushel et al., 1997). Protractions shown in Figures 12, 13, 15, and 16 are, therefore, much weaker than they would be in intact animals. Additionally, it has been hypothesized that retractions during biting consist of the return of the radula to a neutral position as is shown in Figure 14 (Weiss et al., 1986). Currently this is only a hypothesis; however, even in reduced preparations the radula cannot be easily visualized in the neutral state because it is within the buccal mass. In our experiments, in which we monitor radula movements, we find that carbachol commonly elicits a motor program in which the radula retracts slightly beyond its rest position. Although this might suggest that current conceptualizations of biting need to be reevaluated, this issue would obviously be most effectively addressed in experiments in which radula movements are monitored in intact animals. In summary, we use biting-like simply as a relative term to contrast biting-like motor programs with programs in which the radula does retract to reach the esophagus, referred to as swallowing-like (described below).

Simultaneous recordings from left and right B51s revealed that they did receive a $15-20 \mathrm{mV}$ depolarization during biting-like motor programs (Figs. 12, left, 13, middle panel). This depolarization was recorded during the closing/retraction phase of behavior, which is the phase of behavior in which the I4 muscle complex contracts, and will presumably generate centripetal activity in B51.

\section{Centripetal activity in B51 is more likely to occur during swallowing-like motor programs than it is during biting-like motor programs}

Although B51 is centrally depolarized during biting-like motor programs, not many action potentials are recorded from its soma (Fig. 12, left, 13, middle panel). Thus, it is likely that little if any centripetal activity is generated under these conditions (alterna- 
A1

B44

\section{Buccal Nerves Intact}

44



2 All Buccal Nerves Have Been Cut

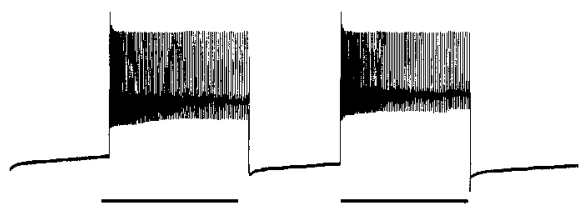

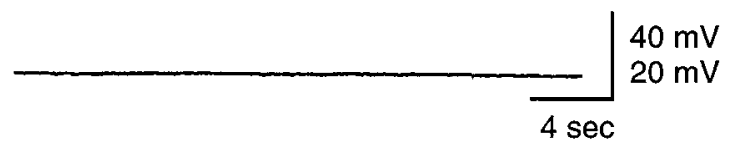

B1

2 Ganglion in 0 Ca; $10 \mathrm{mM}$ Co
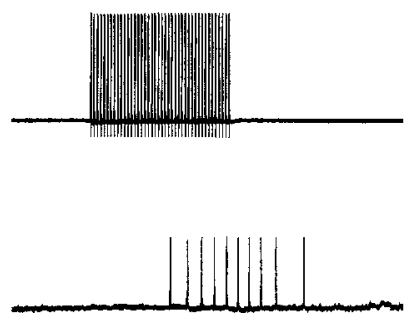

C1
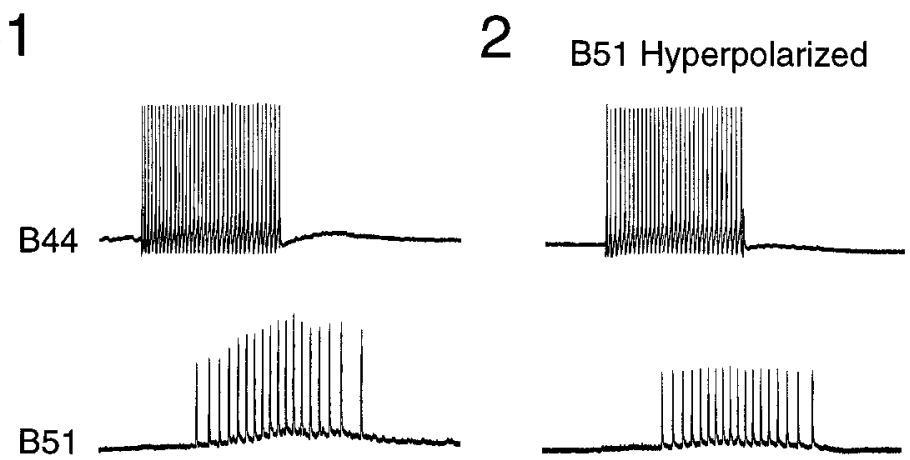

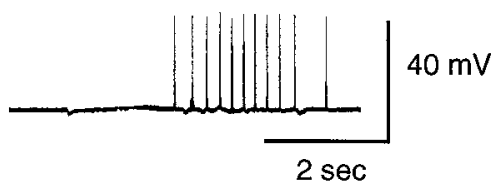

3 Wash

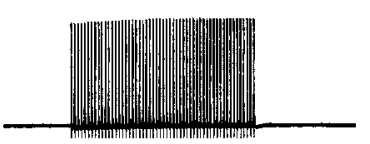

3
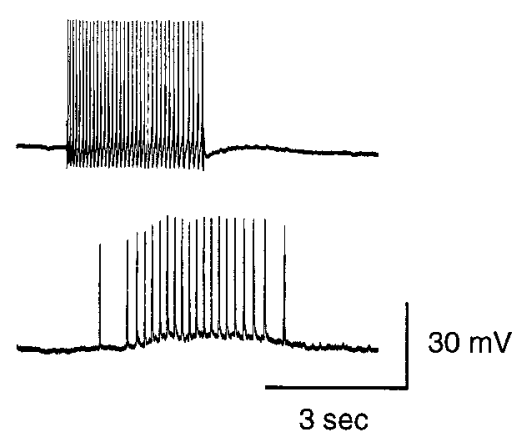

Figure 7. When B44 is stimulated, B51 is activated centripetally. Experiments were conducted in a reduced preparation in which the I4 muscle was attached to a semi-isotonic force transducer, which served as a movement detector. A1, When B44 is stimulated (top), a contraction is elicited in the $\mathrm{I} 4$ muscle (middle), and spikes are recorded in B51 (bottom). Note that responses in B51 are not recorded until a contraction has been elicited and that activity in B51 outlasts the B44 stimulation. A2, The same preparation is shown after the buccal nerves have been cut. Note that B44 stimulation no longer triggers responses in B51. $B$, An experiment in a preparation in which the periphery and buccal ganglion are pharmacologically isolated is shown. In $B 1$ and $B 3$, the periphery and buccal ganglion are both in normal ASW. In B2, the ganglion is in a solution that blocks synaptic transmission. Note that responses in B51 are recorded even when transmission in the buccal ganglion does not occur. $C$, Hyperpolarization reduces the size of spikes evoked in $\mathrm{B} 51$ in response to stimulation of $\mathrm{B} 44$ ( $C 2$ vs $C 1$ or $C 2$ vs $C 3)$.

tively centripetal activity could be generated but spikes could be blocked so that they are not apparent in the B51 soma). As described above, carbachol most commonly elicits a biting-like motor program. In other cases, however, the radula clearly retracted vigorously beyond the rest position and reached the esophagus when carbachol was applied ( $n=4$; Fig. 15). We describe these motor programs as swallowing-like. In other preparations, it was more difficult to classify movements clearly as biting-like or swallowing-like $(n=3)$. During swallowing-like motor programs, spikes are recorded in B51. This is consistent with the idea that centripetal activity is more likely to be generated in B51 under these conditions.

Data obtained in reduced preparations also indicate that centripetal activity in B51 is increased when the resistance to backward rotation of the radula is increased (Fig. $8 B$ ). Under physiological conditions, resistance to backward rotation is likely to be 




B

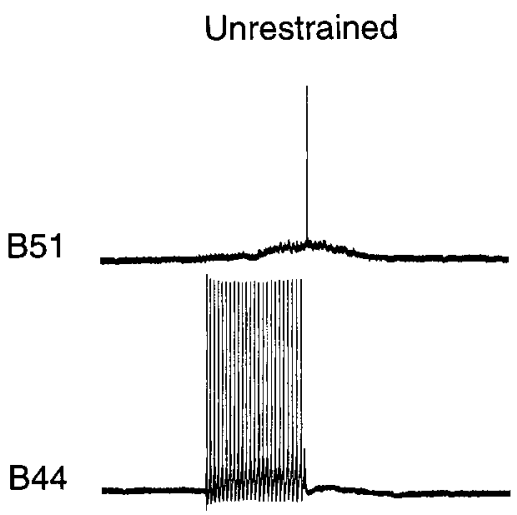

C

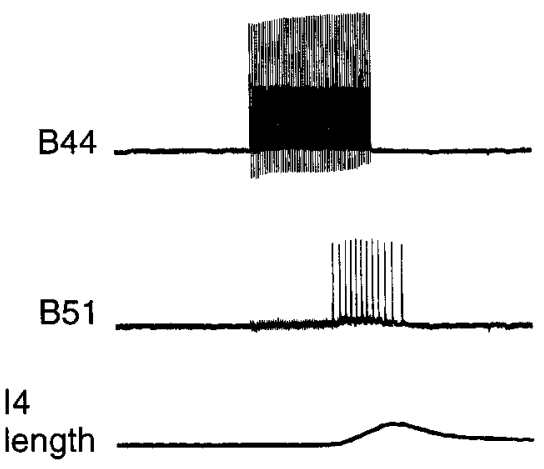

$12 \mathrm{~Hz}$

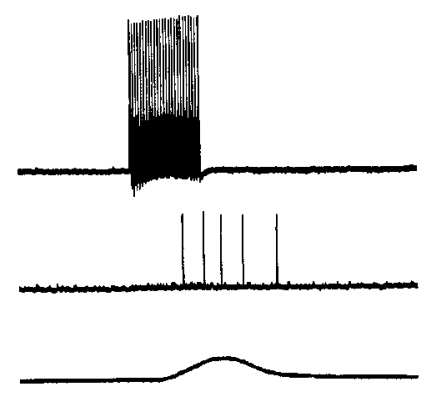

Restrained

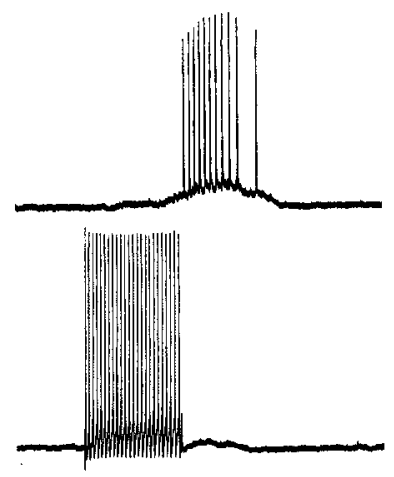

Increased

counterweight


$40 \mathrm{mV}$

$40 \mathrm{mV}$

Figure 8. B51 is a proprioceptor. A, Experiment conducted in a reduced preparation in which the I4 muscle is attached to a movement transducer. Increases in the firing frequency of B44 (top) evoke bigger I4 contractions (bottom) and more centripetal spikes in B51 (middle). B, Experiment conducted in an intact buccal mass preparation. When the radula is restrained, more centripetal responses are recorded in B51. $C$, Experiment conducted in a reduced preparation in which the I4 muscle is counterweighted, i.e., a washer is placed on the free end of the transducer arm. Increasing the counterweight increases the number of centripetal spikes in B51 (middle vs left or middle vs right panels).

increased when food is ingested. Thus, we might expect that centripetal activity would be particularly pronounced in B51 during swallowing responses when an object is pulled into the buccal cavity. To test this possibility, we elicited biting-like motor programs with carbachol, and a piece of polyethylene (PE) tubing was placed between the radula halves as the radula protracted.
When the tubing was successfully grasped between the radula halves and pulled toward the esophagus $(n=2$; Fig. 16), spiking in B51 was indeed enhanced. Although our measurements do not directly indicate whether individual spikes were generated peripherally or centrally in B51, at least some of the increased activity seems to have been generated peripherally because it 


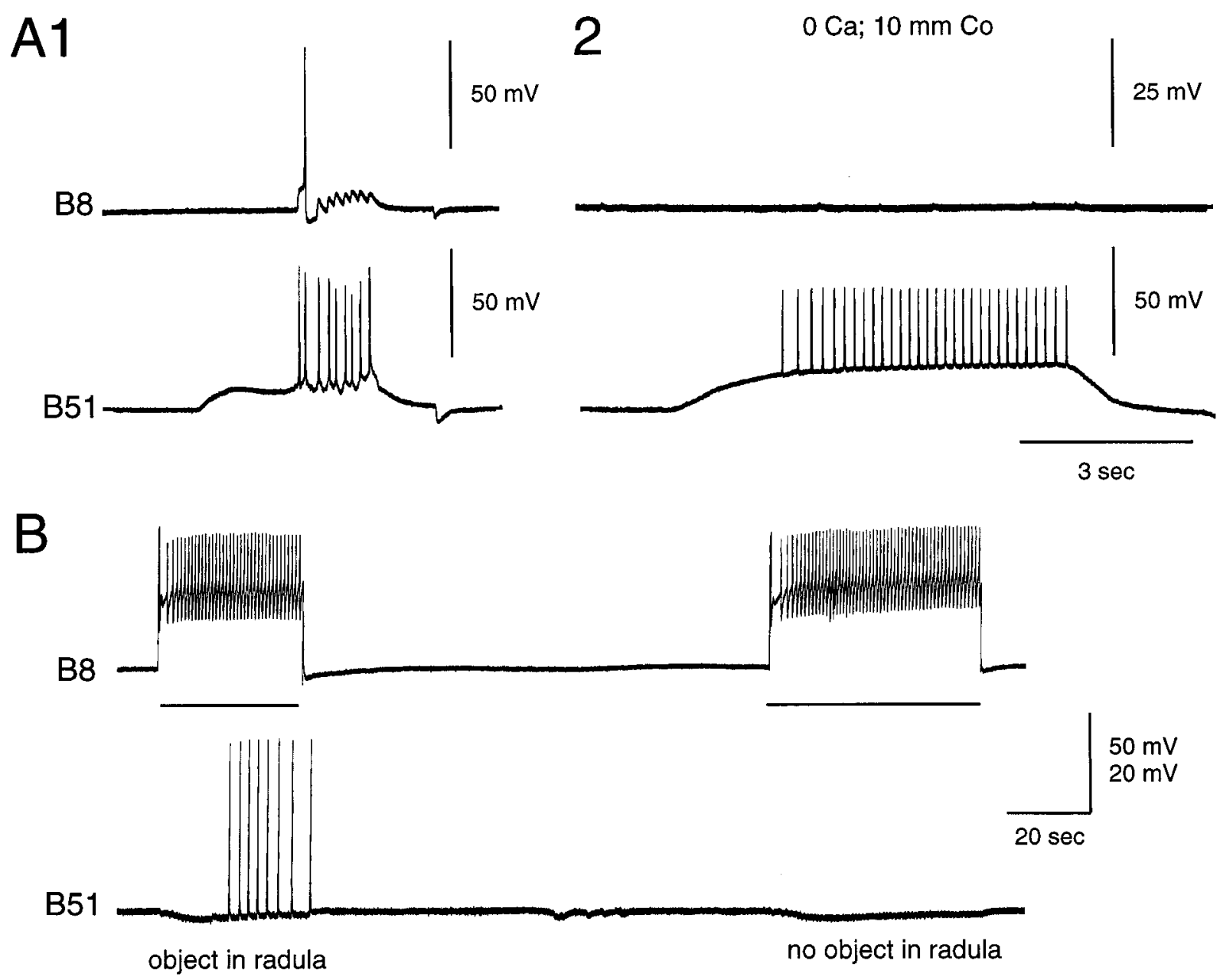

Figure 9. A1, Experiment performed in an isolated buccal ganglion shows that PSPs in B8 are evoked by injection of current into the soma of B51. $A 2$, PSPs are abolished when the buccal ganglion is placed in a solution that abolishes synaptic transmission (i.e., $0 \mathrm{Ca}^{+2}$ and $_{10} \mathrm{~mm} \mathrm{Co}^{+2}$ ). $B$, Experiment conducted in an intact buccal mass preparation is shown. In this experiment B8 activity did not elicit centripetal responses in B51 unless an object was placed between the radula halves.

occurs after B51 is no longer centrally depolarized (see Fig. 16, inset). These findings are, therefore, consistent with the idea that centripetal activity in B51 is enhanced when an object is pulled into the buccal mass.

\section{Depolarization of B51 enhances radula movements during ingestive motor programs}

We next sought to determine whether changes in the activity of B51 are in fact likely to have an impact on buccal motor programs. Thus, although B51 often does not fire at a high frequency during biting-like or swallowing-like motor programs, it is electrically coupled to a number of its follower neurons. Consequently, the magnitude of the rhythmic depolarizations in B51 could play an important role in determining the firing frequency of follower motor neurons. In a similar vein, the duration of the rhythmic depolarizations in B51 could determine the duration of bursts of activity in follower motor neurons. Because the feeding muscles that have been characterized are nonspiking, burst duration generally will affect both contraction duration and amplitude (e.g., Cohen et al., 1978; Evans et al., 1996).

To determine whether changes in the activity of B51 alter feeding motor programs, we elicited rhythmic activity with carbachol and briefly depolarized or hyperpolarized B51. B51 was depolarized in preparations in which radula retractions were relatively weak (e.g., Figs. 12, 13) and hyperpolarized in preparations in which radula movements were more vigorous (Fig. 15). When B51 was depolarized, activity of other radula closing/ retraction neurons was immediately enhanced, and movements of the peripheral musculature were immediately changed ( $n=8$ out of 9 preparations; Fig. 12, 13). Specifically, the radula moved further beyond the rest position (Figs. 12, 13). These "hyperretractions" ceased when the current injection was turned off. When B51 was hyperpolarized, the opposite was observed; e.g., retractions became less vigorous $(n=5$ out of 6 preparations; Fig. 15). These data suggest that changes in B51 activity will produce changes in radula movements during rhythmic motor programs. They also provide insight into the possible functional significance of centripetal activity in B51 in that they show that when activity in B51 is enhanced, as it will be by centripetal activation, the drive to the radula closing/retraction circuitry will be enhanced.

\section{DISCUSSION}

\section{B51 as a pattern-generating interneuron?}

The data of Plummer and Kirk (1990) suggested that B51 is part of the feeding CPG. They showed that B51 receives cyclic input 


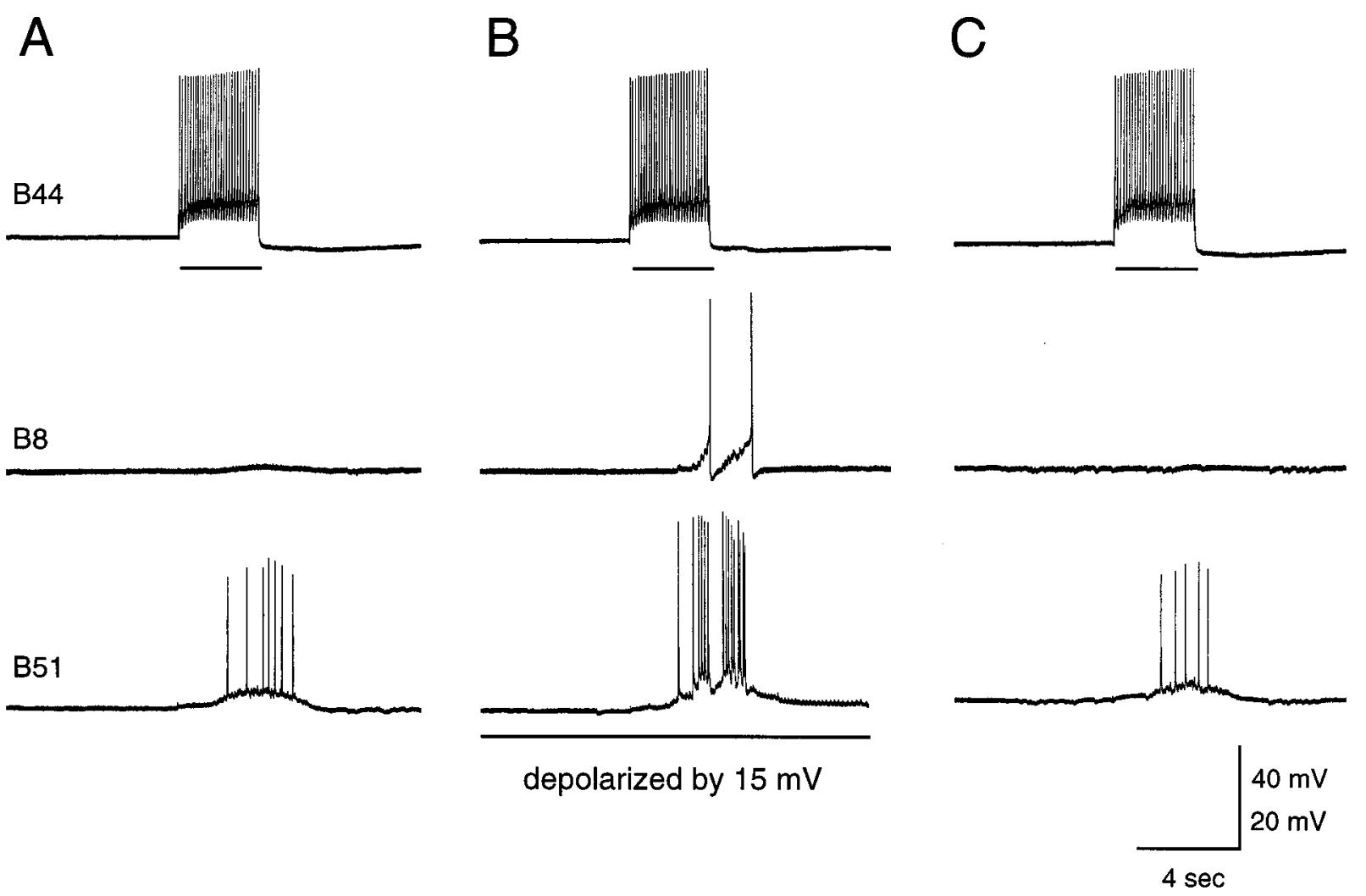

Figure 10. Central depolarization gates afferent activity in B51. Horizontal bars indicate current injection into B44. A, Centripetal spikes in B51 do not elicit PSPs in B8 when B8 is at its resting membrane potential. $B$, Current has been injected into the soma of B51, which has been depolarized by 15 $\mathrm{mV}$. Centripetal spikes now elicit PSPs in B8. $C$, B51 is again at its resting membrane potential. The size of the current pulse injected into B44 is decreased in $B$ so that the B44 firing frequency will remain relatively unchanged; i.e., because B51 and B44 are electrically coupled, some of the current injected into B51 in B is seen by B44. A smaller current pulse is, therefore, used to drive B44 when B51 is depolarized.

during spontaneous and nerve-shock-evoked motor programs. Moreover, depolarization of B51 can initiate bursting in many cells, and when buccal programs cycle spontaneously, depolarization of B51 will phase-shift activity and entrain it. Because methods for inducing ingestive motor programs had, however, not been developed, Plummer and Kirk (1990) could not more precisely define the physiological role of B51. In this study we addressed this issue. We showed that depolarization of B51 will produce widespread changes in the activity of the closing/retraction circuitry during biting-like motor programs. We additionally studied the role of B51 in a less conventional manner; i.e., we changed the membrane potential of B51 and measured changes in radula movements. We showed that depolarization of B51 produces an enhancement of radula retraction, whereas hyperpolarization of B51 produces a decrease in radula retraction. Thus, B51 seems to play an important role in generating feeding motor programs. It may be similar to one of the N2 neurons of Lymnaea (Elliott and Benjamin, 1985; Brierley et al., 1997a,b).

It is unlikely, however, that activity in B51 is necessary for closing/retraction. In most cases radula movements were not eliminated when B51 was hyperpolarized. It is possible that hyperpolarizations did not completely block interactions between B51 and the feeding circuitry. However, cerebral neurons that initiate motor programs synapse directly on both buccal motor neurons and premotor neurons (e.g., Rosen et al., 1991; Xin et al., 1996; Perrins and Weiss, 1998). Cerebral input to the closing/ retraction circuitry is, therefore, not specifically funneled into B51. Moreover, another closing/retraction premotor neuron has been identified [i.e., neuron B64 (Hurwitz and Susswein, 1996)]. Thus, although it is unlikely that radula closing/retraction is solely driven by B51, our data show that changes in the activity of B51 can affect this phase of behavior. Changes in centripetal activity in B51 are, therefore, likely to have an impact on feeding motor programs.

\section{B51 as a sensory neuron}

We show that B51 is a proprioceptor. Although proprioceptors have been described in the Aplysia feeding circuitry (e.g., JahanParwar et al., 1983), their role in behavior has not been established. For example, B4 and B5 (Jahan-Parwar et al., 1983) make inhibitory connections with many motor neurons active during radula closing/retraction (Gardner, 1971; Fiore and Meunier, 1979). It has been hypothesized, therefore, that B4 and B5 are part of a reflex by which they are activated when muscles are stretched. When B4 and B5 fire, they would inhibit follower neurons, which would subsequently be activated as a result of postinhibitory rebound (Jahan-Parwar et al., 1983). How this reflex could function during motor programs is, however, unclear. For example, this model implies that B4 and B5 should fire out of phase with inhibited cells. This does not, however, seem to be the case; B4 and B5 fire during the closing/retraction phase of ingestive motor programs (e.g., Rosen et al., 1991; Church and Lloyd, 1994). Thus, although previous studies have shown that there are proprioceptors in Aplysia and other molluscs (e.g., Kater and Rowell, 1973), other studies have not established that sensory 
A

B44

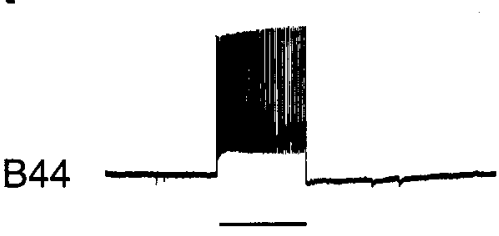

B51
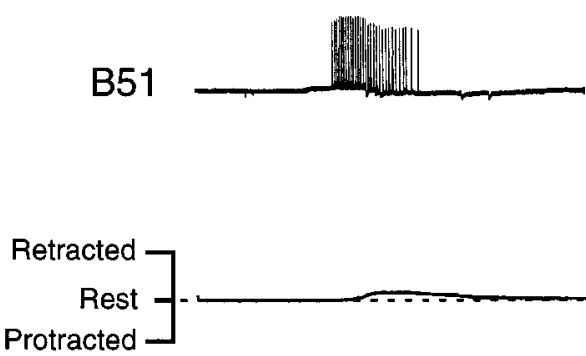
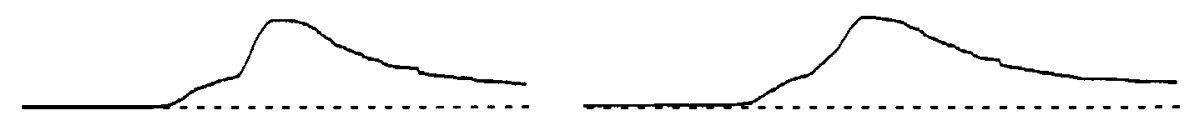

$20 \mathrm{mV}$

B51 depolarized and then released
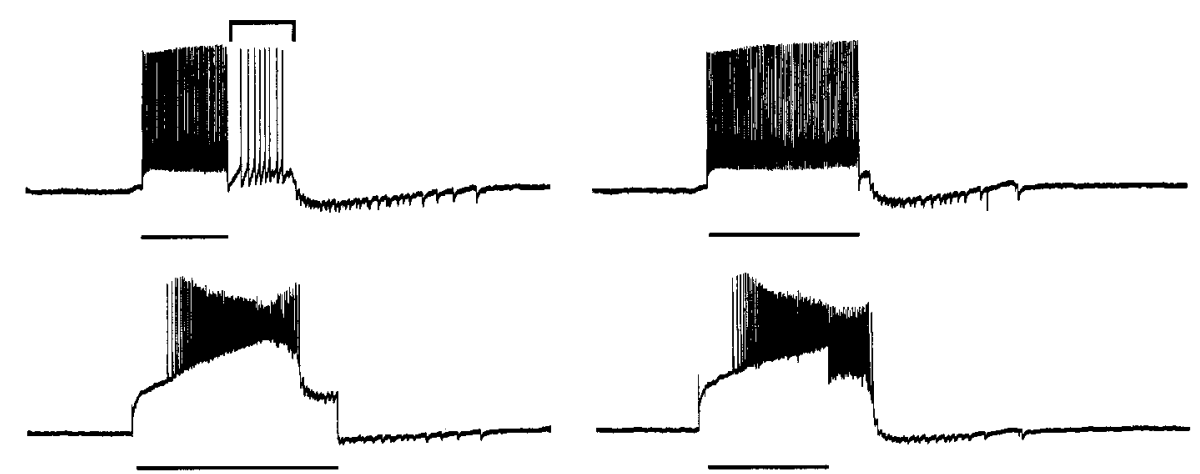

$4 \mathrm{sec}$
B B44

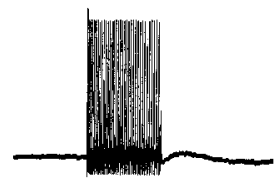

B51

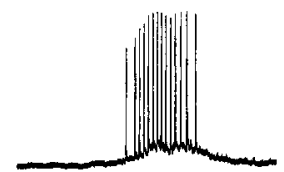

B51 depolarized
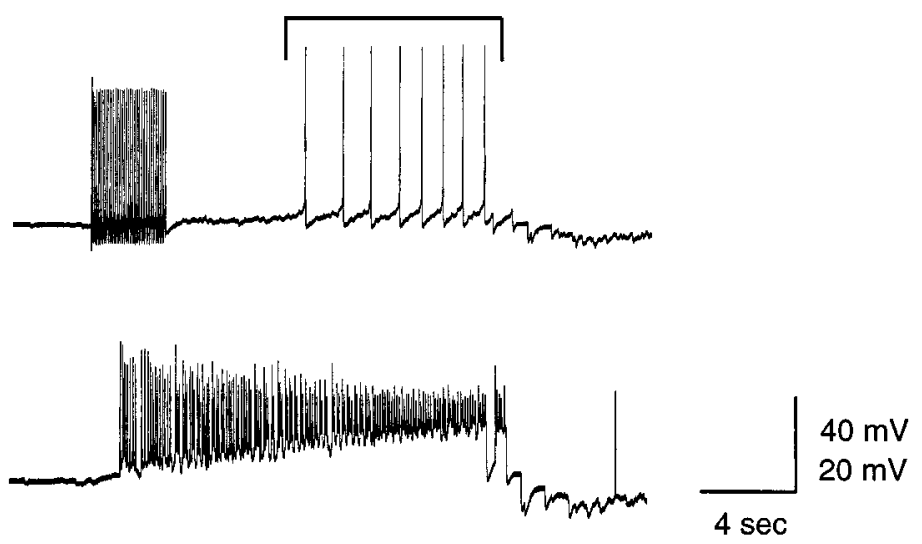

Figure 11. A, Experiment conducted in a semi-intact buccal mass preparation. B44 is depolarized (as indicated by the horizontal bars), and centripetal spikes are elicited in B51. When B51 is at its resting potential (left), these centripetal spikes are not effective at triggering retractions [as shown by the transducer recording (bottom trace on the left)]. When B51 is depolarized (middle, right), however, afferent activity is effective at triggering retractions (bottom traces on the middle and right) and plateau potentials in B51 (middle trace on the right). The increased activity in B51 is effective at driving other neurons. Thus, bracketed action potentials in B44 occur when B44 is no longer being stimulated (top trace in the middle). B, Similar experiment except that B51 is depolarized by $20 \mathrm{mV}$ long before afferent activity is elicited. Although this is a less physiologically relevant manipulation, inward currents activated by the depolarization itself are less likely to be changing when afferent activity is elicited. Under these conditions, depolarization is still effective at "gating" centripetal activity; i.e., when B51 is depolarized, afferent spikes trigger activity in B51 that is more long lasting (bottom trace on right) and is effective at driving other neurons [again bracketed action potentials in B44 occur when current is no longer being injected (top trace on right)].

input is important for the generation of molluscan feeding motor programs. These results also add to the growing body of data that indicate that sensory neurons can be intrinsic parts of CPGs (e.g., Pearson, 1987; Pearson and Ramirez, 1997).

\section{Central gating of afferent activity in B51}

Plummer and Kirk (1990) demonstrated that B51 can generate plateau potentials. Mechanoreceptors that have complex biophysical properties have been described (e.g., Combes et al., 1993, 1995, 1997). This type of arrangement can add to the potential of a sensory neuron for coding peripheral information if intrinsic activity is generated peripherally. For example, the anterior gas- tric receptor of lobster generates bursts of activity in its dendrites (Combes et al., 1993, 1997). Burst frequency, burst duration, and intraburst firing frequency can all be changed by a stimulus (Combes et al., 1997). In contrast, in B51, intrinsic activity only seems to be elicited centrally. Centripetal spikes are generated in a straightforward linear manner.

Because B51 does, however, seem to have such a prominent role in determining the magnitude of motor output, central integration of information in this cell is likely to be more important. We show that centripetal activity in B51 is not effective at eliciting responses in follower neurons unless B51 is simultaneously depolarized centrally. This type of gating has been de- 


\section{B51 Depolarized}
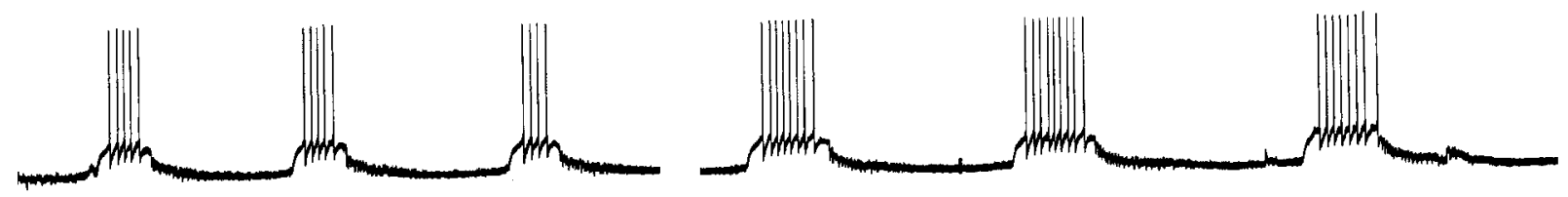

B51
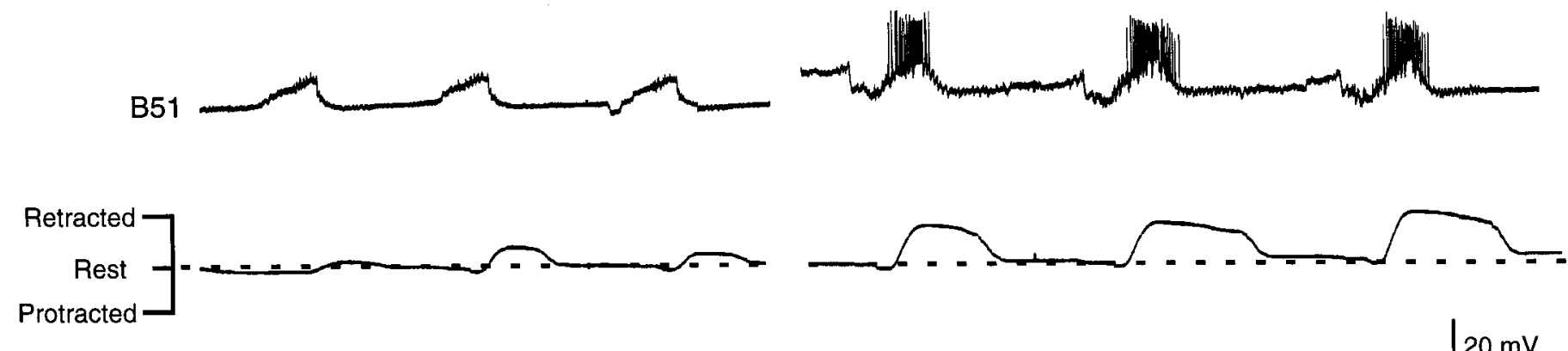

$4 \mathrm{sec}$

Figure 12. Radula movements and intracellular recordings from B15 and B51 during a carbachol-elicited motor program. The fact that B15 is active suggests that these programs are ingestive. In intact animals, B15 does not fire when animals make rejection responses (Cropper et al., 1990). Left, Activity of B15 and B51 without current injection in either neuron. Right, Depolarizing current injected into B51. Note that radula retractions are more vigorous and that activity in B15 remains phase-locked to B51 activity.

\section{B51 depolarized}

\section{B51 depolarized}

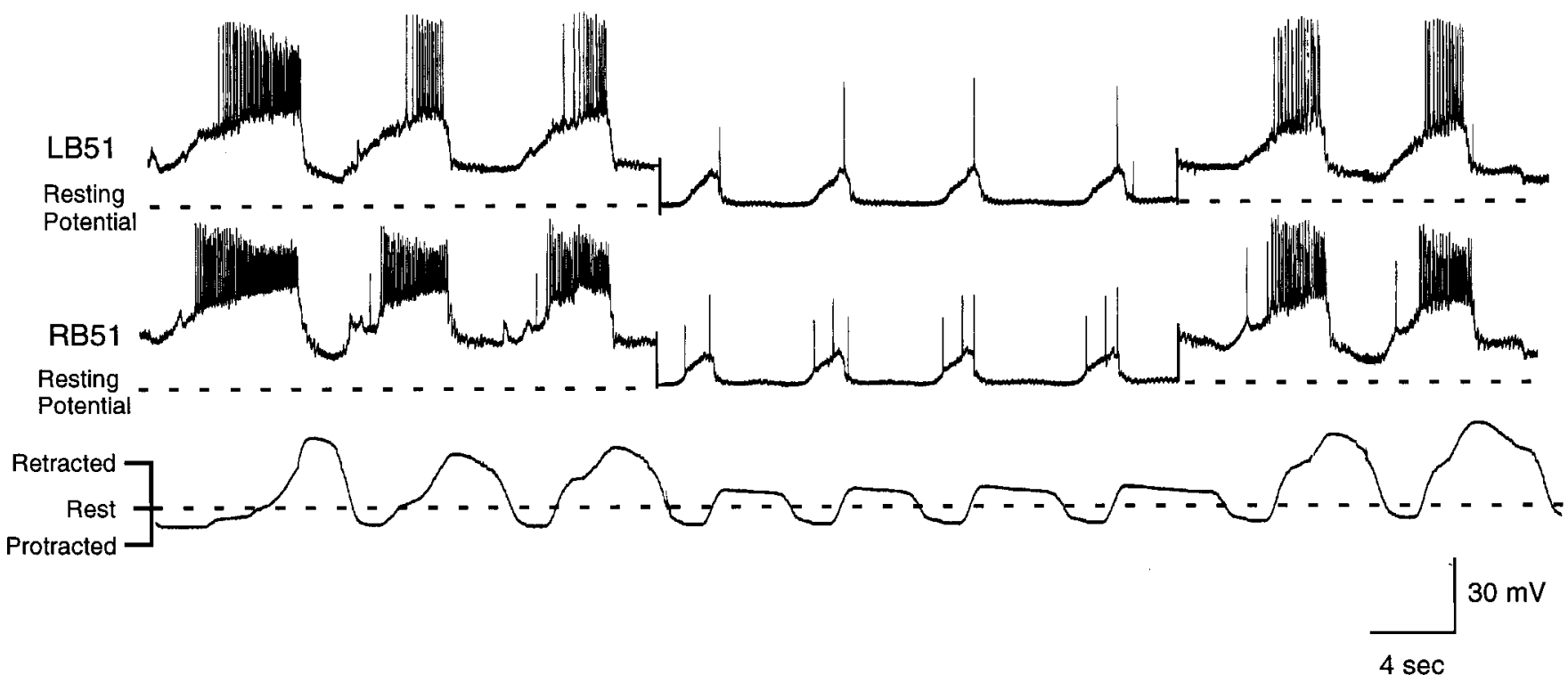

Figure 13. Radula movements and intracellular recordings from left and right B51s during a carbachol-elicited motor program. In this experiment, depolarizing current is periodically injected into both B51s (e.g., left and right panels). The B51s are then returned to the resting membrane potential (e.g., middle panel). Note that when the B51s are depolarized by current injection, CPG-elicited depolarizations are also larger in amplitude and longer in duration. Additionally, radula retractions become more vigorous. Changes in B51 activity and changes in radula movements occur in parallel. Thus, when B51 is no longer depolarized, radula retraction immediately becomes less vigorous. When B51 is depolarized, again radula movements immediately become enhanced.

scribed for other sensory neurons in Aplysia (Rosen et al., 1993, 1994). What specific role the complex biophysical properties of B51 play in integrating central and peripheral information is not yet clear. In otherwise quiescent preparations, we show that when
B51 is depolarized by $15-20 \mathrm{mV}$, as it is during biting-like motor programs, centripetal spikes can cause B51 to exhibit plateau potentials. Although these potentials may not occur under physiological conditions, centripetal activity may at least depolarize 
A

Bite

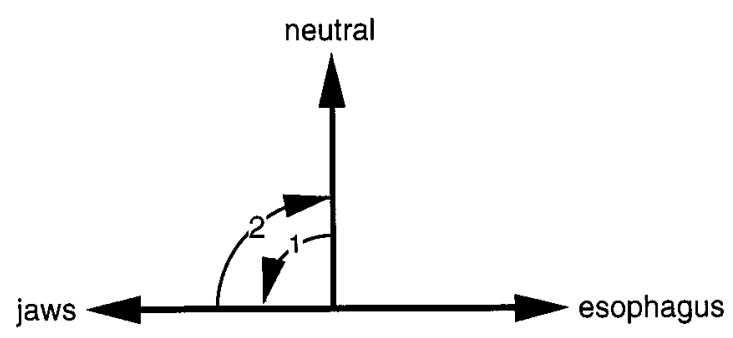

B

\section{Swallow}

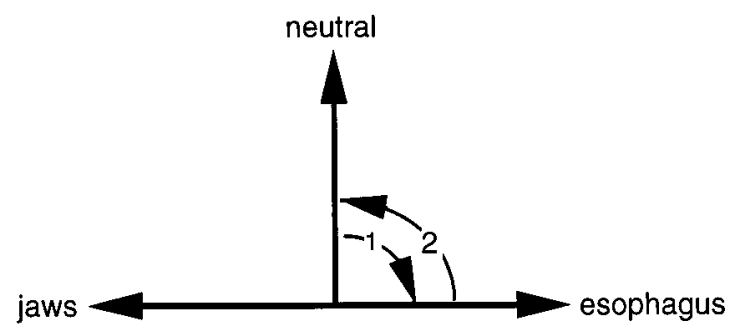

Figure 14. Schematic diagram that illustrates current conceptualizations of how radula movements are thought to change as food is ingested (Weiss et al., 1986). $A$, When food is not ingested, it has been suggested that opening/protraction occurs as the radula moves from a neutral position to a point where it is extended through the animal's open jaws (arrow 1). It has been suggested that the radula then returns to a neutral state (arrow 2). B, When animals make swallowing responses, the radula moves from a rest position to a hyper-retracted position so that food can be deposited in the esophagus (arrow 1). The radula then returns to the rest position (arrow 2).
B51 to the point at which sustained inward currents are partially activated. Effects of these currents on synaptic integration in B51 may be complex (e.g., Simmers and Moulins, 1988a,b).

\section{Physiological role of centripetal activity in B51}

When Aplysia do not successfully ingest food, they bite (Kupfermann, 1974). During biting programs, it is likely that B51 receives a rhythmic central depolarization but is not centripetally activated (Fig. 17). During biting-like motor programs induced by carbachol, spikes are generally not recorded in B51. Additionally, the number of centripetal spikes in B51 is related to the size of I4 muscle contractions and to the degree of resistance that is encountered as the radula rotates backward. I4 muscle contractions will be smaller when animals bite than when they swallow because radula retractions are less vigorous. Because food is not ingested, there also should be little resistance to backward rotation.

When animals do ingest food and swallow, centripetal activity in B51 is likely to be initiated (Fig. 16). This is likely to occur because as food passes through an animal's jaws, it will offer resistance to backward rotation. What will the function of the increased drive of B51 to the retraction circuitry be? It could actually trigger bite to bit-swallow conversions. Thus, food of any size could offer enough resistance to centripetally activate B51, and enhanced B51 activity could solely produce the changes in the activity of the closing/retraction circuitry that are observed when animals ingest food (Cropper et al., 1990). We do not, however, think that this is the case for several reasons. For example, centripetal activity in B51 is only likely to occur toward the end of radula closing/retraction (Fig. 17). B44 starts firing as closing/retraction is initiated, but centripetal activity in B51 occurs with a delay, because it takes time for the I4 muscle to contract. Centripetal activity in B51 is, therefore, likely to occur too late to trigger bite to bite-swallow conversions (see Fig. 16 in which enhanced activity in B44 appears to precede spiking in B51). Moreover, other sensory neurons that are activated by food have been identified, i.e., the radula mechanoafferents B21 and B22 (Rosen et al., 1992; Miller et al., 1994). In contrast to B51, centripetal activity in B21 and B22 is likely to occur early during

\section{B51 hyperpolarized}

B8


Figure 15. Radula movements and intracellular recordings from B51 and B8 during a carbachol-elicited motor program in which radula retractions were relatively vigorous. The B51 neuron was hyperpolarized during the time indicated by the horizontal bar. Note that radula retractions immediately became less vigorous. 

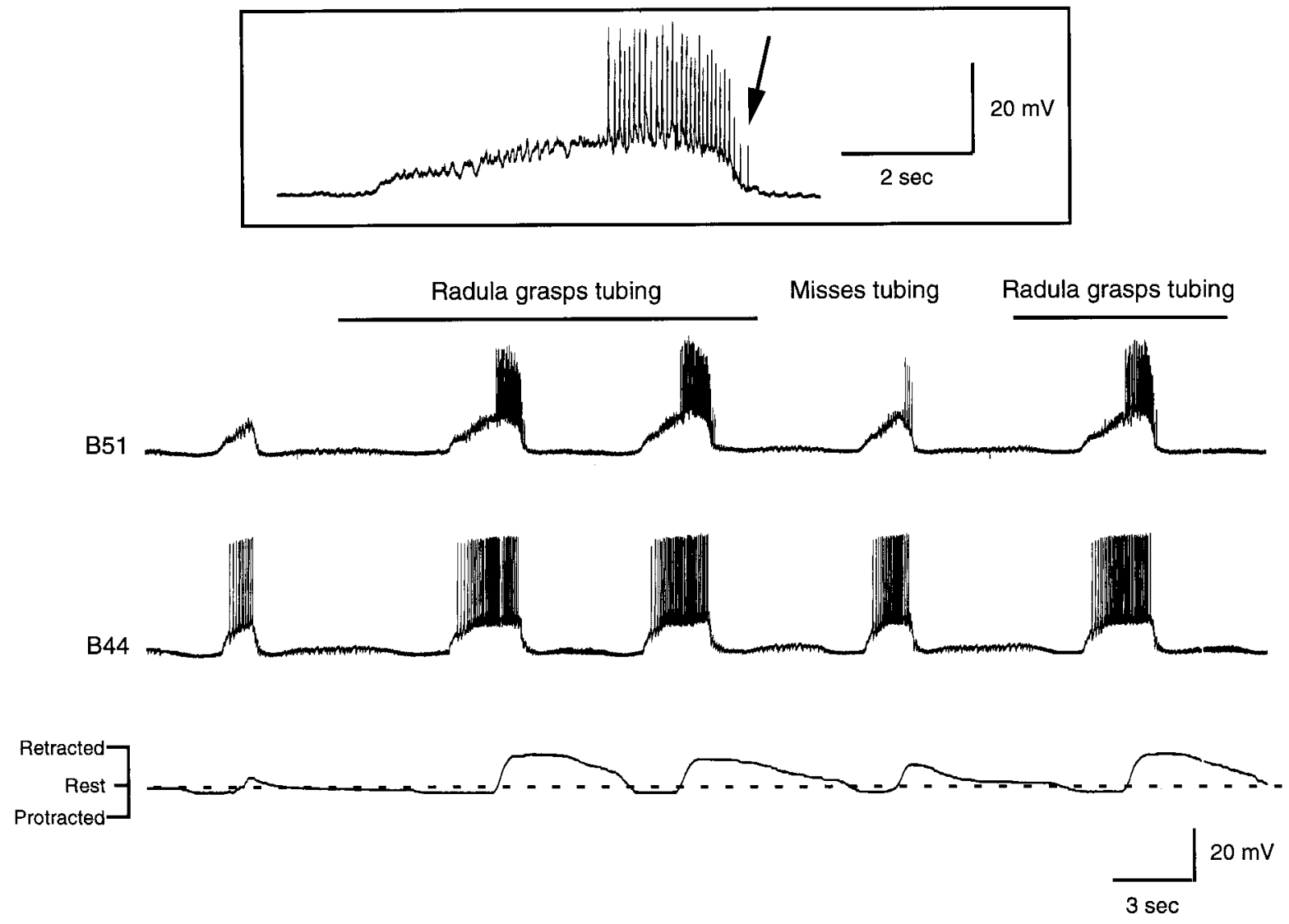

Figure 16. Radula movements and intracellular recordings from B51 and B44 during a carbachol-elicited motor program. At the point indicated by the bar above the top trace, a piece of PE tubing was inserted between the radula halves. Radula retraction became more vigorous, and the piece of PE tubing was pulled through the buccal mass. Activity in B44 and B51 was enhanced in parallel to changes in radula movements. At least some of the increased activity in B51 was likely to have been peripherally generated because spikes are recorded when B51 is no longer centrally depolarized (arrow in record of B51 activity, in inset). Also note that spikes in B51 are of different sizes, which also suggests that they are generated at different sites.

\section{Bite Swallow}

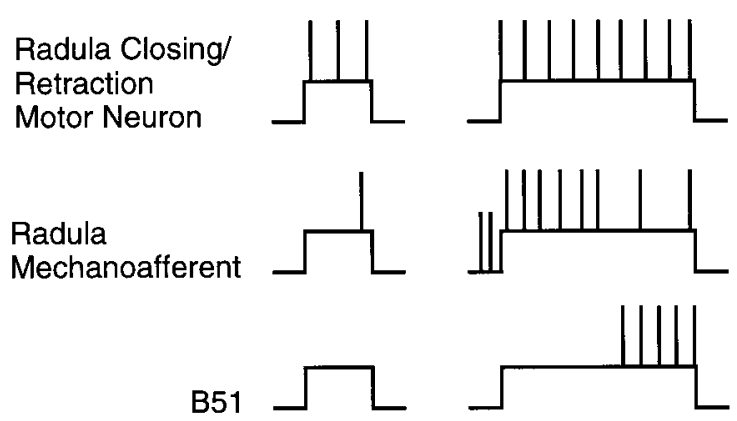

Figure 17. Schematic representation of neural activity during a bite (left) and a swallow (right). When bites are converted to swallows, activity in radula closing/retraction motor neurons is enhanced and prolonged (top). These changes in motor neuron activity result, at least partially, from the activation of buccal sensory neurons. Activity is likely to be initiated in radula mechanoafferents at the end of protraction/opening, as food contacts the maximally extended radula (middle). Radula mechanoafferents are likely to remain active as the radula closes on food and may accommodate with maintained stimulation (Miller et al., 1994). Centripetal activity in B51 is likely to occur relatively late during closing/retraction (bottom). Although B44 starts firing as closing/retraction is initiated, it takes time for the I4 muscle to contract. Radula mechanoafferents may, therefore, trigger bite to bite-swallow conversions, whereas centripetal activity in B51 may be more important as swallows are executed. closing/retraction. Thus, centripetal activity in B51 may not be critical for triggering bite to bite-swallow conversions. Instead it may be important for swallow execution.

Assuming that this is case, an additional question can be asked; namely, how does B51 activity actually affect the magnitude of radula retractions when food is ingested? In our carbachol experiments in which B51 is depolarized, the magnitude of radula retraction is greatly enhanced. Under these conditions, however, an object cannot be moved through the buccal mass in a normal manner because the jaws, etc., are not present and retraction muscles do not have to work against a physiological load. Although we cannot directly assess the physiological impact of B51 on the magnitude of radula retraction in our preparation, data relevant to this issue exist. Namely, experiments in intact animals have indicated that swallows do occur when food is counterweighted (within a certain range) but are not increased in magnitude (Hurwitz and Susswein, 1992). Thus, animals can in fact overcome difficulties to pull food into the esophagus. Presumably, they do this by increasing the drive to the retraction circuitry, e.g., by means of the B51 mechanism. Animals do not, however, increase swallow magnitude. Increases in the activity of the retraction circuitry are, therefore, presumably not important because they make retractions larger than they would have been had resistance not been encountered. Instead they may be important because they allow muscles to cope with the increased load. This makes sense in that for food to be ingested it is simply important 
that it reach the esophagus. Going beyond the esophagus is not likely to be beneficial. Thus, it is possible that under physiological conditions, centripetal activity in B51 functions in a compensatory manner.

In conclusion, B51 is a proprioceptor yet is also a centrally located neuron whose activity strongly impacts buccal motor programs. Consequently, ingestive motor programs are likely to be automatically adjusted to insure that food will be ingested if a reasonable amount of resistance is encountered.

\section{REFERENCES}

Brierley MJ, Staras K, Benjamin PR (1997a) Behavioral function of glutamatergic interneurons in the feeding system of Lymnaea: plateauing properties and synaptic connections with motor neurons. J Neurophysiol 78:3386-3395.

Brierley MJ, Yeoman MS, Benjamin PR (1997b) Glutamatergic N2v cells are central pattern generator interneurons of the Lymnaea feeding system: new model for rhythm generation. J Neurophysiol 78:3396-3407.

Church PJ, Lloyd PE (1991) Expression of diverse neuropeptide cotransmitters by identified motor neurons in Aplysia. J Neurosci 11:618-624.

Church PJ, Lloyd PE (1994) Activity of multiple identified motor neurons recorded intracellularly during evoked feedinglike motor programs in Aplysia. J Neurophysiol 72:1794-1809.

Cohen JL, Weiss KR, Kupfermann I (1978) Motor control of buccal muscles in Aplysia. J Neurophysiol 41:157-180.

Combes D, Simmers J, Nonnotte L, Moulins M (1993) Tetrodotoxinsensitive dendritic spiking and control of axonal firing in a lobster mechanoreceptor neurone. J Physiol (Lond) 460:581-602.

Combes D, Simmers J, Moulins M (1995) Structural and functional characterization of a muscle tendon proprioceptor in lobster. J Comp Neurol 363:221-234.

Combes D, Simmers J, Moulins M (1997) Conditional dendritic oscillators in a lobster mechanoreceptor neurone. J Physiol (Lond) 499:161-177.

Cropper EC, Kupfermann I, Weiss KR (1990) Differential firing patterns of the peptide-containing cholinergic motor neurons B15 and B16 during feeding behavior in Aplysia. Brain Res 522:176-179.

Cropper EC, Evans CG, Rosen SC (1996) Multiple mechanisms for peripheral activation of the peptide-containing radula mechanoafferent neurons B21 and B22 of Aplysia. J Neurophysiol 76:1344-1351.

Delcomyn F (1980) Neural basis of rhythmic behavior in animals. Science 210:492-498.

Drushel RF, Neustadter DM, Shallenberger LL, Crago PE, Chiel HJ (1997) The kinematics of swallowing in the buccal mass of Aplysia californica. J Exp Biol 200:735-752.

Elliott CJH, Benjamin PR (1985) Interactions of pattern-generating interneurons controlling feeding in Lymnaea stagnalis. J Neurophysiol 54:1396-1411.

Evans CG, Cropper EC (1997) A sensory function for the premotoneuron B51 of Aplysia. Soc Neurosci Abstr 23:1045.

Evans CG, Rosen S, Kupfermann I, Weiss KR, Cropper EC (1996) Characterization of a radula opener neuromuscular system in Aplysia. J Neurophysiol 76:1267-1281.

Fiore L, Meunier J-M (1979) Synaptic connections and functional organization in Aplysia buccal ganglia. J Neurobiol 10:13-29.

Gardner D (1971) Bilateral symmetry and interneuronal organization in the buccal ganglia of Aplysia. Science 173:550-553.

Hurwitz I, Susswein AJ (1992) Adaptation of feeding sequences in Aplysia oculifera to changes in the load and width of food. J Exp Biol 166:215-235.

Hurwitz I, Susswein AJ (1996) B64, a newly identified central pattern generator element producing a phase switch from protraction to retraction in buccal motor programs of Aplysia californica. J Neurophysiol 75:1327-1344.

Jahan-Parwar B, Wilson AH, Fredman SM (1983) Role of proprioceptive reflexes in control of feeding muscles of Aplysia. J Neurophysiol 49:1469-1480.

Kandel ER, Tauc L (1966) Anomalous rectification in the metacerebral giant cells and its consequences for synaptic transmission. J Physiol (Lond) 183:287-304.

Kater SB, Rowell CH (1973) Integration of sensory and centrally pro- grammed components in generation of cyclical feeding activity of Helisoma trivolvis. J Neurophysiol 36:142-155.

Kupfermann I (1974) Feeding behavior in Aplysia: a simple system for the study of motivation. Behav Biol 10:1-26.

Miller MW, Rosen SC, Schissel SL, Cropper EC, Kupfermann I, Weiss KR (1994) A population of SCP-containing neurons in the buccal ganglion of Aplysia are radula mechanoafferents and receive excitation of central origin. J Neurosci 14:7008-7023.

Morton DW, Chiel HJ (1993a) In vivo buccal nerve activity that distinguishes ingestion from rejection can be used to predict behavioral transitions in Aplysia. J Comp Physiol [A] 172:17-32.

Morton DW, Chiel HJ (1993b) The timing of activity in motor neurons that produce radula movements distinguishes ingestion from rejection in Aplysia. J Comp Physiol [A] 173:519-536.

Pearson KG (1987) Central pattern generation: a concept under scrutiny. In: Advances in physiological research, pp 167-185. New York: Plenum.

Pearson KG, Ramirez JM (1997) Sensory modulation of patterngenerating circuits. In: Neurons, networks, and motor behavior, pp 225-235. Cambridge, MA: MIT.

Perrins R, Weiss KR (1998) Compartmentalization of information processing in an Aplysia feeding circuit interneuron through membrane properties and synaptic interactions. J Neurosci 18:3977-3989.

Plummer MR, Kirk MD (1990) Premotor neurons B51 and B52 in the buccal ganglia of Aplysia californica: synaptic connections, effects on ongoing motor rhythms, and peptide modulation. J Neurophysiol 63:539-558.

Rao G, Barnes CG, McNaughton BL (1986) Intracellular fluorescent staining with carboxyfluorescein: a rapid and reliable method for quantifying dye-coupling in mammalian central nervous system. J Neurosci Methods 16:251-263.

Rosen SC, Teyke T, Miller MW, Weiss KR, Kupfermann I (1991) Identification and characterization of cerebral-to-buccal interneurons implicated in the control of motor programs associated with feeding in Aplysia. J Neurosci 11:3630-3655.

Rosen SC, Miller MW, Weiss KR, Kupfermann I (1992) SCPcontaining radula mechanoafferent neurons in the buccal ganglion of Aplysia: synaptic connectivity of identified cells. Soc Neurosci Abstr 18:1279.

Rosen SC, Miller MW, Weiss KR, Kupfermann I (1993) Different forms of gating of a peptidergic mechanoafferent neuron by central pattern generator neurons in the feeding system of Aplysia. Soc Neurosci Abstr 19:1700.

Rosen SC, Miller MW, Cropper EC, Kupfermann I (1994) Modulation of outputs of a mechanoafferent neuron by sensory, motor, and interneuronal elements in the feeding pattern generator network of Aplysia. Soc Neurosci Abstr 20:23.

Scott ML, Govind CK, Kirk MD (1991) Neuromuscular organization of the buccal system in Aplysia californica. J Comp Neurol 312:207-222.

Simmers J, Moulins MA (1988a) Disynaptic sensorimotor pathway in the lobster stomatogastric system. J Neurophysiol 59:740-756.

Simmers J, Moulins MA (1988b) Nonlinear interneuronal properties underlie integrative flexibility in a lobster disynaptic sensorimotor pathway. J Neurophysiol 59:757-777.

Steinberg TH, Newman AS, Swanson JA, Silverstein SC (1987) Macrophages possess probenecid-inhibitable organic anion transporters that remove fluorescent dyes from the cytoplasmic matrix. J Cell Biol 105:2695-2702.

Susswein AJ, Rosen SC, Gapon S, Kupfermann I (1996) Characterization of buccal motor programs elicited by a cholinergic agonist applied to the cerebral ganglion of Aplysia californica. J Comp Physiol [A] 179:509-524.

Weiss KR, Chiel HJ, Koch U, Kupfermann I (1986) Activity of an identified histaminergic neuron, and its possible role in arousal in feeding behavior in semi-intact Aplysia. J Neurosci 6:2403-2415.

Wolf H, Pearson KG (1988) Proprioceptive input patterns elevator activity in the locust flight system. J Neurophysiol 59:1831-1853.

Xin Y, Rosen SC, Perrins R, Hurwitz I, Weiss KR, Kupfermann I (1996) Two pairs of the cerebral to buccal interneurons that modulate buccal motor programs in Aplysia are myomodulin peptide-containing cells. Soc Neurosci Abstr 22:2044.

Yang JF, Stein RB, James KB (1991) Contribution of peripheral afferents to the activation of the soleus muscle during walking in humans. Exp Brain Res 87:679-687. 